\title{
Diabetic LDL inhibits cell-cycle progression via STAT5B and $\mathbf{p}^{21}$ waf
}

\author{
Maria Felice Brizzi, ${ }^{1}$ Patrizia Dentelli, ${ }^{1}$ Marzia Pavan, ${ }^{1}$ Arturo Rosso, ${ }^{1}$ Roberto Gambino, ${ }^{1}$ \\ Maria Grazia De Cesaris, ${ }^{2}$ Giovanni Garbarino, ${ }^{1}$ Giovanni Camussi, ${ }^{1}$ Gianfranco Pagano, ${ }^{1}$ \\ and Luigi Pegoraro ${ }^{1}$
}

${ }^{1}$ Dipartimento di Medicina Interna Università di Torino, Torino, Italy

${ }^{2}$ Dipartimento di Scienze Mediche, Università Piemonte Orientale, Novara, Italy

Address correspondence to: Luigi Pegoraro, Department of Internal Medicine, University of Torino, Cso. Dogliotti 14, 10126, Torino, Italy. Phone: 0039-011-6335-539; Fax: 0039-011-6637-520; E-mail: luigi.pegoraro@unito.it.

Received for publication June 26, 2001, and accepted in revised form November 6, 2001.

\begin{abstract}
Modified LDL is a major cause of injury to the endothelium in diabetes. In the present study, we analyzed the effects on endothelial cells of LDL recovered from type 2 diabetic patients (dm-LDL) or from nondiabetic subjects (n-LDL). Treatment of human umbilical vein endothelial cells with dm-LDL, but not n-LDL, led to the accumulation of cells in G1. To dissect the molecular mechanisms of this effect, we analyzed the expression and function of the cyclin-dependent kinase inhibitor $\mathrm{p} 21^{\text {waf }}$, a cell cycle regulator known to be a target of the signal transducers and activators of transcription (STATs). $\mathrm{dm}$-LDL led to transient STAT5 phosphorylation and the formation of a STAT5-containing complex and activated p21 $1^{\text {waf }}$ expression at the transcriptional level. Expression of the dominant-negative form of STAT5B, but not of STAT5A, significantly decreased both $\mathrm{p} 21^{\text {waf }}$ expression and the fraction of cells in G1. Finally, immunofluorescence analysis demonstrated that activated STAT5 is expressed in newly formed intraplaque vessels and in endothelial cells lining the luminal side of the plaque. Similarly, $\mathrm{p} 21^{\mathrm{waf}}$ immunoreactivity was found in the neointimal vasculature. Our results suggest a role of STAT5B as a regulator of gene expression in diabetes-associated vascular disease.
\end{abstract}

J. Clin. Invest. 109:111-119 (2002). DOI:10.1172/JCI200213617.

\section{Introduction}

Atherosclerosis is a multistep chronic inflammatory disease that involves the interplay between soluble mediators and vascular/inflammatory cells (1). Multiple risk factors, including diabetes, are involved in this pathological process $(2,3)$. Several lines of evidence indicate that either qualitative or quantitative abnormalities of LDL in the diabetic setting may contribute to its atherogenicity, and in particular to the macrovascular complications that it causes (4-7). Although clinically significant complications of atherosclerosis, such as plaque ulceration, rupture, and thrombosis occur in established atherosclerotic lesions, understanding of the mechanisms of early lesion formation offers the possibility to delay or prevent further progression. A select set of transcriptional factors may be critical in both the initiation and the expansion of lesions. One of these, NF- $\mathrm{KB}$, has been linked to the onset of atherosclerosis (8). It has been reported that both native and modified LDL activate a series of NF-KB-dependent genes that are relevant to the pathophysiology of the vessel wall (8). A similar set of genes is known to be a target of the signal transducers and activators of transcription (STATs) (9). STATs are a family of latent cytoplasmic proteins that, upon activation, acquire DNA-binding activity, translocate into the nucleus, bind to specific promoter elements, and control the expression of target genes (9). Two different but highly homologous STAT5 genes have been isolated, and defined as STAT5A and STAT5B (10). These STAT proteins undergo activation in response to different stimuli, and exert transcriptional activation on a number of genes that are involved mainly in the control of cell proliferation (11).

A large body of evidence indicates that the cell cycle is controlled by a series of regulatory molecules known as cyclins, cyclin-dependent kinases (Cdk's), and Cdk inhibitors (CKIs) (12). Among these latter molecules are p21 $1^{\text {waf }}$ (13) and p27kip1 (14), dual inhibitors of Cdk's and of the replication factor known as proliferating cell nuclear antigen (PCNA). The effects of $\mathrm{p} 21^{\text {waf }}$ and p27kip1 lead to cell-cycle arrest and inhibition of DNA replication, respectively (15-17). Evidence is accumulating that supports the idea that certain regulators of cell proliferation modulate cyclin/Cdk activity by affecting CKI expression (18-20). Recent data also support the role of $\mathrm{p} 21^{\text {waf }}$ in mediating inhibition of cell proliferation that is associated with laminar shear stress (21). Although no data are available on the molecular mechanisms involved in shear stress-induced $\mathrm{p} 21^{\text {waf }}$ expression, it has been reported that $\mathrm{p} 21^{\text {waf }}$ is a target of STAT5 during megakaryocytic differentiation (22).

The vast majority of studies aimed at elucidating the role of LDL in diabetes-associated vascular complications have been performed with artificially glycated and/or oxidized LDL (ox-LDL) (23). The aim of the present study was to evaluate the effects of the natural 
a

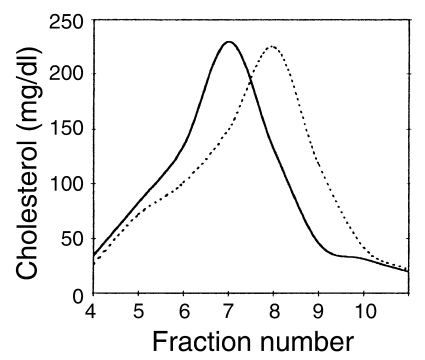

b

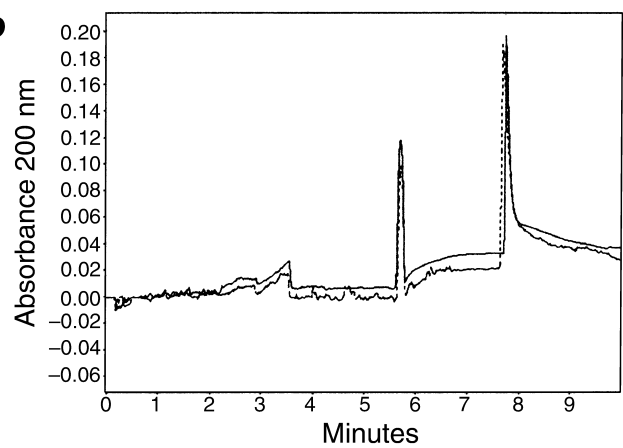

Figure 1

Lipid profile of $\mathrm{n}$-LDL and dm-LDL. (a) Density-gradient ultracentrifugation LDL profile of two representative subjects. The dotted line represents a diabetic patient and the solid line a control sample. Cholesterol profiles are expressed as concentration $(\mathrm{mg} / \mathrm{dl})$ in each fraction. (b) Electropherogram of purified LDL. The dotted line represents $d m-L D L$ and the black line represents $n-L D L$. Peak 1 is the electroendosmotic marker; peak 2 is purified LDL.

plasma constituent recovered from type 2 diabetic patients $(\mathrm{dm}-\mathrm{LDL})$ on endothelial cells. We found that $\mathrm{dm}$-LDL affects cell-cycle progression via STAT5Bmediated $\mathrm{p} 21^{\text {waf }}$ induction. Moreover, activated STAT5 and $\mathrm{p} 21^{\text {waf }}$ immunoreactivity was present in human intraplaque neovessels.

\section{Methods}

Patients and controls. Blood was withdrawn from nine blood donors and from nine type 2 diabetic patients in bad metabolic control (fasting plasma glucose $\geq 10 \mathrm{mM}$ and hemoglobin $A 1 c \geq 10 \%)$. None of them was under insulin, and all were treated with sulphonylurea agents. The mean age was $68 \pm 4$ years in the diabetic group, and $62 \pm 3$ years in controls. Each group consisted of five men and four women.

Reagents. M199 medium (endotoxin tested), BSA, and protein A-Sepharose were from Sigma Chemical Co. (St. Louis, Missouri, USA). Bovine calf serum (endotoxin tested) was obtained from HyClone Laboratories Inc. (Logan, Utah, USA). Trypsin was purchased from Difco Laboratories Inc. (Detroit, Michigan, USA). Nitrocellulose filters, horseradish peroxidase-conjugated protein $\mathrm{A}$, the molecular weight markers $\left[\alpha-{ }^{32} \mathrm{P}\right] \mathrm{dCTP}$, $\left[\gamma_{-32}{ }^{32}\right]$ ATP, and $\left[\alpha-{ }^{32} \mathrm{P}\right]$ UTP, the chemiluminescence reagent enhanced, and the Poly(dIdC):poly(dIdC) were obtained from Amersham Pharmacia Biotech Italia (Milano, Italy). Histone H1 was from Roche Diagnostics S.P.A. (Monza, Italy). The presence of endotoxin contamination of LDL preparations was tested by the Limulus amebocyte assay; the concentration was 0.05 ng/ml. Anti-STAT5A (L-20), anti-STAT5B (G-2 and C-17), anti-p21waf, and anti-p27 kip1 antisera were obtained from Santa Cruz Biotechnology Inc. (Heidelberg, Germany). Anti-phospho-STAT5, anti-CD105, and anti-CD45 were from New England Biolabs Inc. (Beverly, Massachusetts, USA).

Isolation, characterization, and oxidation of $L D L$. Blood was centrifuged for 15 minutes at $12,000 \mathrm{~g}$ at $4^{\circ} \mathrm{C}$. LDL was separated according to the method of Redgrave and Carlson (24). Oxidation of n-LDL was performed in a cell-free system as described (25). Oxidation was confirmed by agarose gel electrophoresis. Purity of LDL was assessed by capillary electrophoresis at $200 \mathrm{~nm}$.

Thiobarbituric acid-reactive substances assay and capillary electrophoresis. LDL subfractions were pooled, and peroxidation was measured using a thiobarbituric acid-reactive substances (TBARS) assay (26). TBARS concentrations were calculated from a calibration curve that was prepared using 1,1,3,3-tetramethoxypropane as a standard. Diene conjugate quantification was performed by capillary electrophoresis as described (27). LDL mobility in capillary electrophoresis was calculated as described by Cruzado et al. (28).

Cell cultures and transfections. HUVECs were isolated as previously described (29). ECV304 cells were transfected with the dominant-negative STAT5A ( $\triangle$ STAT5A) and STAT5B $(\triangle$ STAT5B) constructs (11) by the lipofectin method. Expression of the $\triangle$ STAT5 proteins was analyzed by Western blotting with an anti-STAT5 antibody, and the positive clones were tested by electrophoretic mobility shift assay (30).

Flow cytometry. HUVECs or ECV304 cells were stimulated in serum-free medium with n-LDL $(100 \mu \mathrm{g} / \mathrm{ml})$ or $\mathrm{dm}-\mathrm{LDL}(100 \mu \mathrm{g} / \mathrm{ml})$ for 12 hours, and then fixed with $70 \%$ ethanol. After digestion with RNase, DNA was stained with propidium iodide and analyzed with a flow cytometer.

Western blot analysis and immunoprecipitation studies. HUVEC monolayers were processed as described (30) and incubated with or without $100 \mu \mathrm{g} / \mathrm{ml}$ of either nLDL or dm-LDL for the indicated time periods. Protein concentrations of cell lysates were obtained and samples were eluted and processed as previously described (30).

Preparation of nuclear extracts and gel retardation assay. Nuclear extracts from untreated or LDL-treated HUVECs were prepared as described by Sadowski and Gilman (31). The double-stranded p21SIE2 oligonucleotide sequence $5^{\prime}$-GATCCTTTCTGAGAAATGG-3' (22) was used. Gel retardation reactions were performed as previously described (30).

Cdk2 kinase assay. Cdk2 immunoprecipitated from dmLDL-stimulated $(100 \mu \mathrm{g} / \mathrm{ml})$, STAT transfected ECV304 clones was divided into two aliquots. Cdk2 immunoprecipitates were resuspended in kinase buffer consisting of $20 \mathrm{mM}$ Tris/ $\mathrm{HCl}$ (pH7.4), $10 \mathrm{mM} \mathrm{MgCl}_{2}$, and 1 $\mathrm{mM}$ DTT containing $50 \mu \mathrm{M}\left[\gamma^{-32} \mathrm{P}\right] \mathrm{ATP}$ and $5 \mu \mathrm{g} / \mathrm{ml}$ of histone $\mathrm{H} 1$, and incubated for 30 minutes at $30^{\circ} \mathrm{C}$. 
Table 1

Characteristics of $n-L D L$ and dm-LDL

\begin{tabular}{lcccc}
\hline Subject ID & Chol $(\mu \mathrm{g} / \mu \mathrm{l})$ & $\mathrm{ApoB}(\mu \mathrm{g} / \mu \mathrm{l})$ & Chol/apoB & HbA1c \\
$1(\mathrm{~d})$ & 1.61 & 1.26 & 1.3 & $12 \%$ \\
$2(\mathrm{~d})$ & 0.65 & 0.50 & 1.3 & $11.8 \%$ \\
$3(\mathrm{~d})^{\mathrm{A}}$ & 1.50 & 1.06 & 1.4 & $10.8 \%$ \\
$4(\mathrm{~d})^{\mathrm{A}}$ & 1.93 & 1.41 & 1.4 & $10.9 \%$ \\
$5(\mathrm{~d})$ & 1.65 & 1.16 & 1.4 & $12.4 \%$ \\
$6(\mathrm{~d})$ & 2.00 & 1.50 & 1.3 & $13.8 \%$ \\
$7(\mathrm{~d})^{\mathrm{A}}$ & 1.03 & 0.75 & 1.4 & $11.3 \%$ \\
$8(\mathrm{~d})^{\mathrm{A}}$ & 1.10 & 0.81 & 1.35 & $10.1 \%$ \\
$9(\mathrm{~d})$ & 0.71 & 0.49 & 1.4 & $12.1 \%$ \\
$10(\mathrm{n})$ & 0.97 & 0.50 & 1.9 & \\
$11(\mathrm{n})^{\mathrm{A}}$ & 1.04 & 0.61 & 1.7 & \\
$12(\mathrm{n})$ & 1.41 & 0.90 & 1.56 & \\
$13(\mathrm{n})$ & 0.93 & 0.57 & 1.6 & \\
$14(\mathrm{n})^{\mathrm{A}}$ & 1.19 & 0.73 & 1.6 & \\
$15(\mathrm{n})^{\mathrm{A}}$ & 1.66 & 0.98 & 1.7 & \\
$16(n)$ & 1.36 & 0.85 & 1.6 & \\
$17(n)$ & 1.88 & 1.04 & 1.8 & \\
$18(n)^{\mathrm{A}}$ & 0.89 & 0.56 & 1.6 & \\
& & & &
\end{tabular}

HbA1c, hemoglobin A1c; n, n-LDL; d, dm-LDL; chol, cholesterol. AUsed for pilot experiments.

Agarose beads were removed by centrifugation, and the supernatants were fractionated by $8 \%$ SDS-PAGE.

Nuclear run-off transcription. Approximately $2 \times 10^{7}$ HUVECs were stimulated with dm-LDL $(100 \mu \mathrm{g} / \mathrm{ml})$ and lysed in Nonidet P-40 lysis buffer (32). Nuclei were collected and stored as previously described (32). Elongation of nascent RNA was performed as previously described (32). Labeled RNA was purified as described by Gariglio et al. (33), and processed as previously described (32).

Detection of reactive oxygen species. 5,6-caroxy-22, 72dichlorofluorescin-diacetate (DCF-DA) $(20 \mathrm{mM}$ final concentration) was added to HUVECs in the various culture conditions at time 0 . At the times indicated, the cells were subjected to flow cytometric analysis as described (34). TNF- $\alpha$ was used as positive control (35). Immunofluorescence microscopy. Carotid specimens from diabetic or hypercholesterolemic patients and healthy carotid specimens were fixed and embedded in paraffin and processed as described (36). Sections were stained with the anti-phospho-STAT5, the anti-p2 $1^{\text {waf }}$, and the anti-CD105 antibodies. Indirect immunofluorescence analysis was performed as previously described (36).

\section{Results}

LDL preparation and characterization. In diabetic subjects, the highest cholesterol was found in the LDL subfractions with densities ranging from $1.037 \mathrm{~g} / \mathrm{ml}$ to 1.044 $\mathrm{g} / \mathrm{ml}$. In healthy subjects, the highest level of cholesterol was found in the LDL subfractions with densities ranging from $1.028 \mathrm{~g} / \mathrm{ml}$ to $1.035 \mathrm{~g} / \mathrm{ml}$. dm-LDL was consistently smaller and denser than normal LDL. This pattern was confirmed by the density-gradient ultracentrifugation profile (Figure 1a) and by the lower cholesterol/apoB ratio (Table 1). Lipid peroxidation was assessed by TBARS assay and capillary electrophoresis. The results demonstrated the absence of TBARS and of conjugated dienes (determined by measuring absorbance at $234 \mathrm{~nm}$ in capillary electrophoresis) in both n-LDL and dm-LDL preparations. It is widely acknowledged that changes in electrophoretic mobility are the most reliable indicator of LDL modifications (27). Indeed, the results reported in Figure $1 \mathrm{~b}$ demonstrated the same electrophoretic mobility pattern for both n-LDL and dm-LDL. These results, which are consistent with data obtained by Jenkins et al. (37), rule out the possibility that a significant degree of lipid peroxidation was present in the LDL preparations. Moreover, the finding that $\mathrm{dm}-\mathrm{LDL}$ eluted as a single sharp peak (Figure 1b) led us to exclude the presence of copurified substances in our preparations. After characterization of each sample, pilot experiments were performed using single preparations; in subsequent experiments, pooled sera from normal donors or diabetic patients were used.

$d m$-LDL affects cell-cycle progression by regulating $p 21^{\text {waf }}$ gene expression. The effects of $\mathrm{n}-\mathrm{LDL}$ and $\mathrm{dm}-\mathrm{LDL}$ on the cell cycle were evaluated by flow cytometric analysis. The flow cytometric analysis for the DNA content in HUVECs indicated that the cell populations in the $S$ and $\mathrm{G} 2 / \mathrm{M}$ phases decreased after the cells were subjected to $\mathrm{dm}-\mathrm{LDL}$. Indeed, cells in the $\mathrm{S}$ phase were reduced to $8 \%$, of the total compared with the control value of $24.6 \%$ and the $\mathrm{n}-\mathrm{LDL}$ value of $25.5 \%$. Values are for the $\mathrm{S}$ phase only. This effect, induced by $\mathrm{dm}-\mathrm{LDL}$, was accompanied by an increase to $85.8 \%$ in the percentage of cells in G0 or G1 (Figure 2). In order to dis-
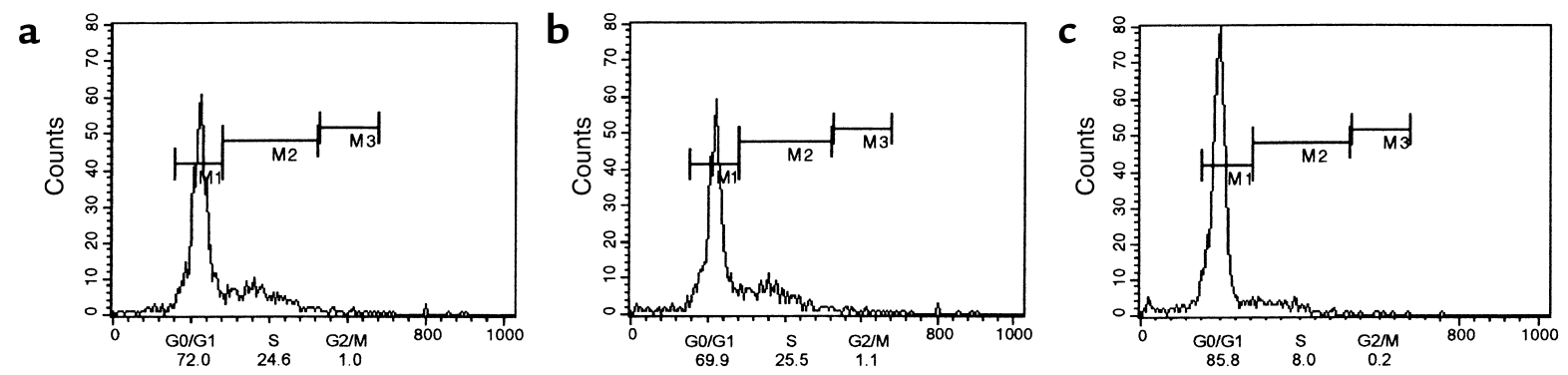

Figure 2

Effects of dm-LDL and n-LDL on the cell cycle. HUVECs, unstimulated (a) or stimulated for 12 hours with n-LDL (b) or dm-LDL (c), were harvested and fixed with ethanol. DNA was stained with propidium iodide, and fluorescence was evaluated by flow cytometry using FACScan equipment (Becton Dickinson Immunocytometry Systems, San Jose, California, USA). Percentage of cells in each phase is shown, as determined by ModFit LT software (Verity Software House Inc., Topsham, Maine, USA). Similar results were obtained in three different individual experiments. 


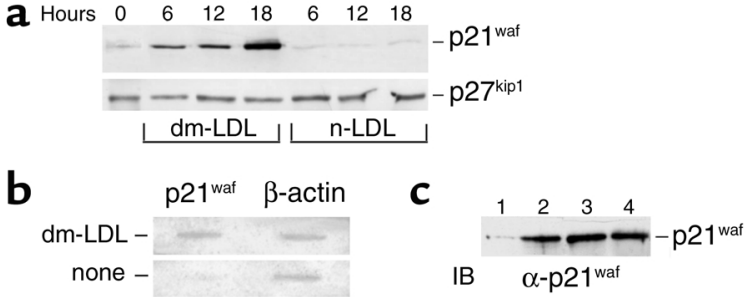

Figure 3

dm-LDL increases p21 waf expression via a transcriptional mechanism. (a) p $21^{\text {waf }}$ and $p 27^{k i p 1}$ expression. Proteins from lysed HUVECs were incubated with $\mathrm{n}$-LDL or dm-LDL for different time periods, subjected to $15 \%$ SDS-PAGE, and electrophoretically transferred to nitrocellulose filters. The filters were immunoblotted with an antip2 $1^{\text {waf }}$ antibody or an anti-p2 $7^{\text {kip } 1}$ antiserum. Five different experiments were performed with similar results. (b) p21waf gene transcription. RNAs were obtained from nuclei prepared from unstimulated HUVECs or HUVECs stimulated with dm-LDL for 90 minutes. Equal cpm of the purified radioactive RNAs from the respective reactions were hybridized against $p 21^{\text {waf }}$ and $\beta$-actin cDNAs dot-blotted on the same filter. Similar results were obtained in two different experiments. (c) n-LDL does not compete with dm-LDL. HUVECs were unstimulated (lane 1) or were stimulated for 18 hours with dm-LDL alone (lane 2 ) or in combination with n-LDL (50- and 100-fold excess, lanes 3 and 4, respectively), and evaluated for $\mathrm{p} 21^{\text {waf }}$ as above. Similar results were obtained in three different experiments. IB, immunoblot.

sect the molecular mechanisms involved in this effect, we analyzed the expression of two CKIs: $\mathrm{p} 21^{\mathrm{waf}}$ and p27Kip1. As shown in Figure 3a, and consistent with flow cytometric analysis, treatment with dm-LDL but not $\mathrm{n}$-LDL led to an increase in $\mathrm{p} 21^{\text {waf }}$ expression. In contrast, the expression of $\mathrm{p} 27^{\mathrm{kip} 1}$ was not affected by either $\mathrm{n}-\mathrm{LDL}$ or $\mathrm{dm}-\mathrm{LDL}$ (Figure $3 \mathrm{a}$ ). Sustained increased levels of $\mathrm{p} 21^{\mathrm{waf}}$ can be mediated by various mechanisms, including increased RNA transcription (12). Therefore, to evaluate whether the mechanism involved in $\mathrm{p} 21^{\text {waf }}$ expression was dependent upon gene transcription, nuclear run-off experiments were performed. As depicted in Figure 3b, while nuclear p2 $1^{\text {waf }}$ mRNA was barely detectable in unstimulated cells, it was clearly induced upon dm-LDL treatment, showing that a transcriptional mechanism regulated $\mathrm{dm}$ LDL-mediated $\mathrm{p} 21^{\text {waf }}$ expression.

It is known that modified LDL may exert its effects by binding to membrane structures other than the canonical LDL receptor $(38,39)$. The possibility that a receptor other than the native LDL receptor was engaged by $\mathrm{dm}$-LDL was indirectly evaluated by competition experiments. HUVECs were stimulated with $\mathrm{dm}$-LDL alone or in combination with 50- and 100fold excesses of $\mathrm{n}-\mathrm{LDL}$, and then assayed for $\mathrm{p} 21^{\mathrm{waf}}$ expression. As shown in Figure 3c, n-LDL was unable to abrogate the effect of $\mathrm{dm}-\mathrm{LDL}$ on $\mathrm{p} 21^{\text {waf }}$ expression, suggesting that a receptor other than the natural LDL receptor is recruited by $\mathrm{dm}-\mathrm{LDL}$.

$d m-L D L$ induces reactive oxygen species production. It is known that modified LDL-induced signaling involves intracellular reactive oxygen species (ROS) production
(40). Accordingly, we examined the effects of n-LDL and $\mathrm{dm}-\mathrm{LDL}$ on intracellular ROS production. As shown in Figure 4, dm-LDL, but not n-LDL, induced a burst of ROS that peaked at 15 minutes as measured by DCF fluorescence. TNF- $\alpha$ was used as a positive control. Similar results were obtained with the lucigeninenhanced chemiluminescence assay using a 1251 LKB luminometer (Perkin-Elmer Instruments, Bad-Wildbad, Germany) (data not shown).

$d m$-LDL triggers STAT5 activation. STAT5A and STAT5B are pleiotropic regulators of many genes, including p21 waf (22). To assess the role of the STAT5 pathway in regulating dm-LDL-mediated $21^{\text {waf }}$ expression, the effect of dm-LDL on STAT5 activation was analyzed. The results shown in Figure 5a demonstrate that, unlike n-LDL, dm-LDL was able to trigger STAT5 tyrosine phosphorylation. It is known that hydrogen peroxide is able to activate STAT1 and STAT3 (41). However, neither n-LDL nor dm-LDL was able to induce STAT3 activation (data not shown), and when ox-LDL was used to stimulate HUVECs, no STAT5 activation could be detected (Figure 5b). To assess whether $\mathrm{dm}$-LDL-elicited STAT5 activation was involved in p21 waf induction, an electrophoretic mobility shift assay was performed using the p21SIE2 sequence, which is a potential STAT binding site in the $\mathrm{p} 21^{\text {waf }}$ promoter region (22). As shown in Figure 5c, nuclear extracts from dm-LDL-treated, but not from untreated or n-LDL-treated, endothelial cells were able to form a p21SIE2-binding complex that was competed for by an added excess of unlabeled p21SIE2 probe. The presence of STAT5 in the DNA-protein complex induced by $\mathrm{dm}$-LDL was demonstrated by the ability of the antibody to STAT5 to supershift the p21SIE2-binding complex (Figure 5d). Consistent with the inability of dm-LDL to trigger STAT3 phosphorylation, the antibody to STAT3 did not supershift the $\mathrm{dm}$ LDL-induced p21SIE2-binding complex (Figure 5d).

Expression of $\triangle S T A T 5$ proteins in the ECV304 cell line. Modified forms of STAT5 acting as dominant-negative proteins were obtained by removing most of the

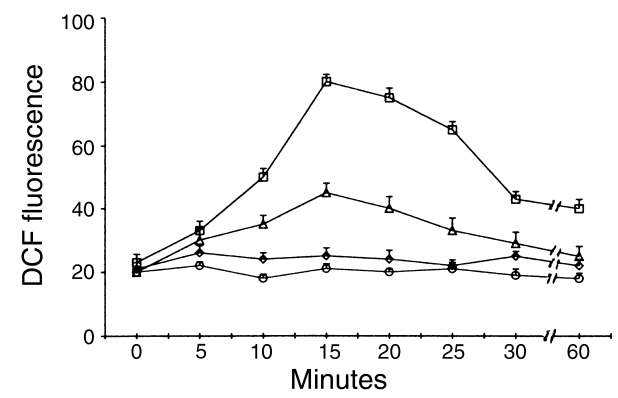

\section{Figure 4}

Kinetics of dm-LDL-induced ROS production. DCF was added to unstimulated HUVECs (circles) or HUVECs stimulated with n-LDL (diamonds), dm-LDL (triangles), or TNF- $\alpha$ (squares). DCF fluorescence was measured over a 60-minute period. Time 0 represents basal DCF fluorescence. The results are the mean of three individual experiments, each performed in duplicate. 
C-terminal tyrosines (11). To assess the biological relevance of STAT5 protein activation in dm-LDL-mediated $\mathrm{p} 21^{\text {waf }}$ expression, $\triangle$ STAT5A and $\triangle$ STAT5B constructs were used to transfect ECV304 cells. The ECV304 cell line, recently shown to be identical to the T24 cell line derived from a human bladder carcinoma (42), shares several features with endothelial cells (43, $44)$, including the expression of receptors for native and modified LDL (45). Moreover, in our preliminary experiments, ECV304 cells and HUVECs exhibited a similar response to dm-LDL (data not shown). Cells expressing the $\triangle$ STAT5 proteins were selected on the basis of their ability to inhibit activation of endogenous STAT5 proteins. To this end, two stable clones denoted as $\triangle$ STAT5A and $\triangle$ STAT5B, and a clone expressing the Neo vector were stimulated with IL-3, which is able to activate both STAT5 proteins in these cells (M.F. Brizzi, unpublished data). As shown in Figure $6 a, \Delta$ STAT5 expression affected the ability of IL-3 to trigger the activation of endogenous STAT5. In contrast, IL-3 triggered STAT5 tyrosine phosphorylation in Neo-expressing cells.

Expression of $\triangle S T A T 5 B$, but not $\triangle S T A T 5 A$, drastically reduces $d m-L D L-$ mediated $p 21^{\text {waf }}$ expression. To assess whether the effect of dm-LDL on $\mathrm{p} 21$ waf expression was directly mediated by STAT5, the two different $\triangle$ STAT5 clones were stimulated for 18 hours with dm-LDL or nLDL, and p21 waf expression was assayed. Figure $6 \mathrm{~b}$ shows that only in $\triangle$ STAT5B-expressing cells, not in Neo vector-expressing or $\triangle$ STAT5A-expressing cells, $\mathrm{dm}$-LDL failed to induce $\mathrm{p} 21^{\text {waf }}$ expression. The presence of endogenous STAT5B may explain the increased level of $\mathrm{p} 21^{\text {waf }}$ observed in the $\triangle$ STAT5A clone. Moreover, the role of STAT5B in regulating $\mathrm{p} 21^{\text {waf }}$ expression was confirmed by the observation that no DNA-binding activity could be detected in the nuclear extract from the dm-LDL-stimulated $\triangle$ STAT5B clone (Figure 6c). Expression of $\triangle S T A T 5 B$ rescues $C d k 2$ activity and prevents accumulation of cells in G1. p21 waf is known to regulate Cdk2 activity (12). The finding that dm-LDL was unable to upregulate $221^{\text {waf }}$ in the $\Delta$ STAT5B-expressing cells led us to investigate whether this effect was associated with an increase in Cdk2 activity. To this end, Cdk2 kinase assays of cells expressing the different constructs were performed. As shown in Figure 6d, consistent with the role of $\mathrm{p} 21^{\text {waf }}$ in regulating Cdk2 activity, we found that, upon dm-LDL treatment, Cdk2 activity was restored by transfection of cells with $\triangle$ STAT5B but not with $\triangle$ STAT5A or Neo vector.

The role of STAT5B in regulating cell-cycle arrest in response to dm-LDL was further examined. Ectopically transfected ECV304 cells were stimulated with $\mathrm{dm}$-LDL, and assessed for their ability to progress in the cell cycle. In cultures of cells expressing the Neo or the $\triangle$ STAT5A construct, treatment with $\mathrm{dm}$-LDL led to a marked reduction of the percentage of cells in $\mathrm{S}$ phase, and to an increase in the percentage of cells in G0 or G1 (Figure 7). In contrast, dm-LDL treatment of cells expressing the $\triangle$ STAT5B construct led to an increase in the percentage of cells in S phase (40.9\%) and to a corresponding reduction in the percentage of cells in G0 or G1. These observations indicate that increased $\mathrm{p} 21^{\text {waf }}$ expression and the resulting decrease in Cdk2 activity may account for $\mathrm{dm}$-LDL-mediated cell-cycle arrest.

Endothelial cells from atherosclerotic plaques are immunore-

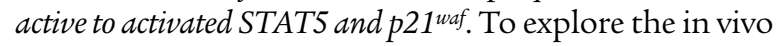
expression of activated STAT5 and $\mathrm{p} 21^{\text {waf }}$, nonatherosclerotic human carotid specimens and atherosclerot-
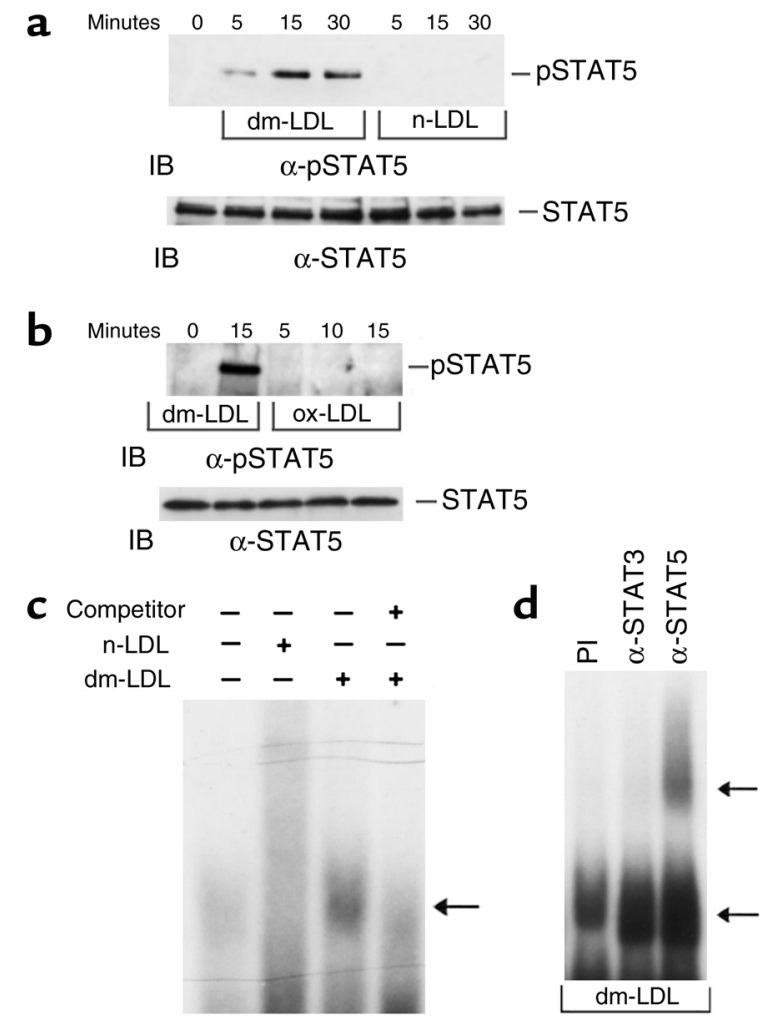

\section{Figure 5}

STAT5 phosphorylation and p21SIE2 binding activity are triggered by dm-LDL but not ox-LDL. (a) Kinetics of STAT5 phosphorylation. Starved HUVECs were stimulated with dm-LDL or $n-L D L$ for the indicated times. After undergoing SDS-PAGE, lysed proteins were electrophoretically transferred to a nitrocellulose filter, immunoblotted, and photographed. (b) ox-LDL does not activate STAT5. HUVECs processed as above were stimulated with dm-LDL (15 minutes) or ox-LDL for the indicated times. Proteins from lysed HUVECs were processed as above. The filters were immunoblotted with anti-phospho-STAT5 antibody and reprobed with anti-STAT5 antiserum. Four individual experiments were performed with similar results. Unphosphorylated and phosphorylated STAT5 are indicated. (c) p21SIE2 complex formation. Nuclear extracts were prepared from HUVECs stimulated for 30 minutes with $\mathrm{n}-\mathrm{LDL}$ or $\mathrm{dm}$-LDL. Competition was performed by addition of a 50-fold excess of unlabeled p21SIE2 probe (competitor). The DNA-protein complexes were resolved by nondenaturing PAGE. (d) The p21SIE2-binding complex is antigenically related to STAT5 but not to STAT3. Nuclear extracts from dmLDL-treated HUVECs were preincubated with an anti-STAT5 or antiSTAT3 antibody as indicated, and the DNA-protein complexes were resolved. Arrows indicate the p21SIE2 complex (lower arrow) and the supershifted species (upper arrow). 


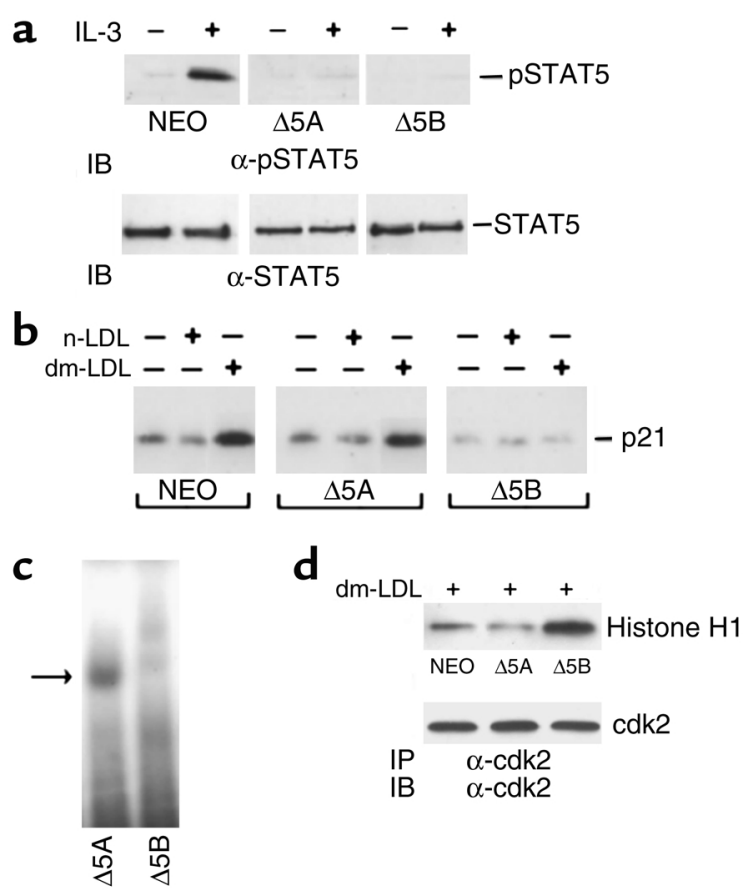

Figure 6

Effect of the $\triangle$ STAT5s on $\mathrm{p} 21^{\text {waf }}$ expression, p21SIE2-binding activity, and Cdk2 kinase activity. (a) Effect of dominant-negative expression on endogenous STAT5. Cell extracts from ECV304 cells transfected with Neo vector (NEO), $\triangle$ STAT5A $(\Delta 5 \mathrm{~A})$, or $\triangle \mathrm{STAT5B}(\Delta 5 \mathrm{~B})$ cDNAs were stimulated for 10 minutes with IL-3 $(10 \mathrm{ng} / \mathrm{ml})$. Cell extracts were subjected to SDS-PAGE and processed as above. The filters were immunoblotted with anti-phospho-STAT5 and reprobed with an anti-STAT5 antiserum. Similar results were obtained in three different experiments. (b) Effect of $\triangle$ STAT5 constructs on p21 waf expression. Cells transfected with the different constructs were unstimulated (-) or stimulated for 18 hours (+) with n-LDL or dmLDL. Cell extracts were processed, and the filters were immunoblotted with an anti-p21 waf antiserum. (c) DNA-protein complex formation. Nuclear extracts from cells expressing $\triangle$ STAT5A or $\triangle$ STAT5B were stimulated with dm-LDL, processed, and resolved. The p21SIE2 complex is indicated. (d) Effect of p21 waf expression on Cdk2-associated histone $\mathrm{H} 1$ kinase activity. Cells expressing Neo, $\triangle$ STAT5A, or $\triangle$ STAT5B cDNAs were stimulated with dm-LDL for 18 hours. Cell lysates were immunoprecipitated with an anti-Cdk2 antiserum and divided into two aliquots. One aliquot was subjected to an in vitro kinase assay (upper panel), fractionated by SDS-PAGE, and subjected to autoradiography. The second aliquot was subjected to SDSPAGE and transferred to a nitrocellulose filter, and the filter was immunoblotted with an anti-Cdk2 antibody. Three different experiments were performed with similar results. IP, immunoprecipitation.

ic plaques from human carotid arteries were analyzed for activated STAT5. The images shown in Figure 8, $\mathrm{a}-\mathrm{d}$, demonstrate that both endothelial cells lining the intraplaque vessels and the plaque luminal side, and smooth muscle cells from media express activated STAT5. No STAT5 activation was found in endothelial cells lining nonatherosclerotic vessels, while only a few fibers in the media were positive. Similarly, a positive immunoreactivity for $\mathrm{p} 21^{\mathrm{waf}}$ was found in intraplaque neovessels, but not in other intraplaque cellular components (Figure 8, e-g).

\section{Discussion}

Regulation of the cell cycle is achieved through a complex and ordered sequence of events controlled by Cdk's, the activation of which depends on their association with protein subunits and the cyclins, and on regulatory phosphorylation (12). The activation of Cdk's is negatively regulated by several CKIs. The four major mammalian CKIs fall into two classes. $\mathrm{p} 21^{\text {waf }}$ and $\mathrm{p} 27^{\mathrm{kip} 1}$ are related proteins with a preference for $\mathrm{Cdk} 2$ and Cdk4-cyclin complexes, whereas $\mathrm{p}^{16^{\mathrm{INK} 4}}$ and $\mathrm{p} 15^{\mathrm{INK} 4 \mathrm{~B}}$ are closely related CKIs that are specific for Cdk4- and Cdk6-cyclin complexes (12). In the present study, we demonstrate that dm-LDL treatment leads to an accumulation of cells in G1 by increasing the level of p21 waf. Although a sustained increase in levels of p2 $1^{\text {waf }}$ resulting from different stimuli could be mediated by various mechanisms (decreased protein degradation, increased mRNA translation rate, or increased mRNA half-life), a major mode of regulation is transcriptional (12). Accordingly, our run-off experiments strongly support a role for transcription in regulating p21 $1^{\text {waf }}$ expression upon dm-LDL stimulation.

p27kip1 was originally identified as a Cdk2 inhibitor whose activity, but not total protein amount, increased throughout the cell cycle (20). This seemed to be associated with the release from intracellular compartments of p27 kip 146,47$)$, and a similar mechanism might account for the stable expression of $\mathrm{p} 27^{\mathrm{kip} 1}$ observed in our experiments.

The members of the STAT family undergo phosphorylation, dimerization, and nuclear translocation to activate target genes (9). Among these, cell cycle-related genes are known to be transcriptionally regulated by STAT5 (48). Moreover, it has been reported that during megakaryocyte differentiation, STAT5 regulates $\mathrm{p} 21^{\text {waf }}$ expression (22). In the present study, we found that dm-LDL, but not n-LDL or ox-LDL, triggers STAT5 activation. Moreover, in nuclear extracts from dmLDL-treated cells, the formation of a STAT5-containing p21SIE2-binding complex suggests that dm-LDL promotes $\mathrm{p} 21^{\text {waf }}$ transcription through STAT5 activation. The molecular mechanisms underlying the activation of the STAT5 pathway by $\mathrm{dm}-\mathrm{LDL}$ remain to be defined. In cytokine- or growth factor-mediated STAT activation, a ligand-dependent phosphorylation of the receptor creates a docking site for the Src homology 2 domain of STAT, thus recruiting STAT into the receptor complex (9). It is known that modified LDL interacts with target cells through different receptors, such as the scavenger receptors (38) and/or the receptor for the advanced glycated end products (39). Indeed, our experiments showing that n-LDL was unable to compete with dm-LDL, to activate STAT5, or to induce ROS production suggest that $\mathrm{n}-\mathrm{LDL}$ and $\mathrm{dm}$-LDL bind to different receptors. Although we were unable to detect an immunoprecipitable complex between STAT5 and scavenger receptors for the advanced glycated end products (data not shown), it is possible that their association occurs either indirectly or through a 

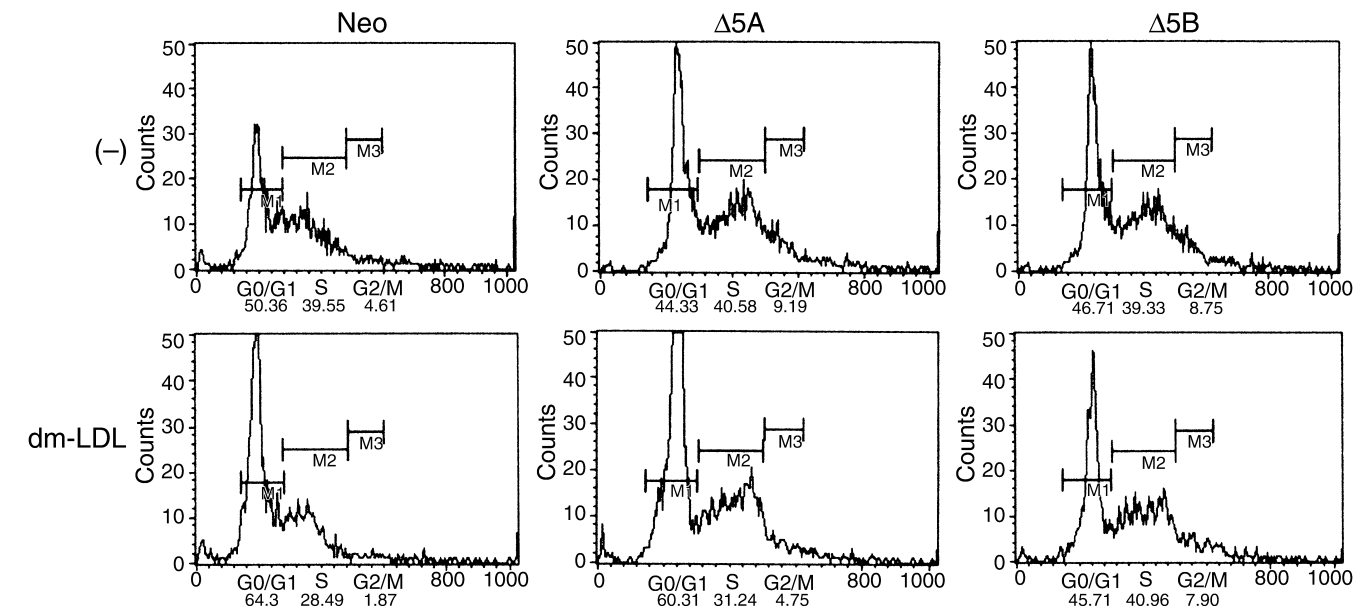

Figure 7

Effects of $\triangle$ STAT5 expression on dm-LDL-mediated cell-cycle events. Neo, $\triangle$ STAT5A, and $\triangle$ STAT5B clones were unstimulated (-) or were stimulated for 12 hours with dm-LDL, harvested, and fixed with ethanol. DNA was stained with propidium iodide, and fluorescence was evaluated by flow cytometry. The percentage of cells in each phase is shown (see legend to Figure 2). Four different experiments were performed with similar results.

low-affinity interaction. However, regardless of the mechanisms and of the receptor engaged by $\mathrm{dm}-\mathrm{LDL}$ to elicit STAT5 activation, the findings that ectopic expression of $\triangle$ STAT5B was able to alter $\mathrm{dm}$ LDL-mediated upregulation of $\mathrm{p} 21^{\text {waf }}$ expression, to rescue Cdk2 activity, and to prevent accumulation of cells in G1 strongly suggests a role for STAT5B in regulating this effect on endothelial cells.

Qualitative changes of LDL, such as glycation and/or oxidation, may account for the increased atherogenic risk in diabetes (2). Indeed, dm-LDL did not show a significant degree of lipid peroxidation, but was characterized by a decreased cholesterol/apoB ratio and by a density profile showing a subfraction distribution corresponding to the LDL subclass defined as pattern B (49). It is commonly accepted that, because of their susceptibility to glycation and/or oxidation, these small, dense particles are responsible for the increased atherogenic risk in diabetes (49). Since dm-LDL did not bind to the canonical LDL receptor, did not activate a STAT3-mediated pathway (as shown by the inability of anti-STAT3 antiserum to modify the mobility shift of the p21SIE2-binding complex), and was recovered from patients in poor metabolic control, it is reasonable to assume that glycation, rather than oxidation, represents the qualitative change in $\mathrm{dm}-\mathrm{LDL}$ that accounts for our results.

In conditions such as atherosclerosis, intimal angiogenesis occurs as part of the adaptive changes known as vasculature remodeling (50). Recent clinical studies suggest that risk factors for coronary artery disease may modify an individual's capacity for angiogenesis and vascular remodeling. Specifically, hypercholesterolemia and diabetes have been shown to be associ-
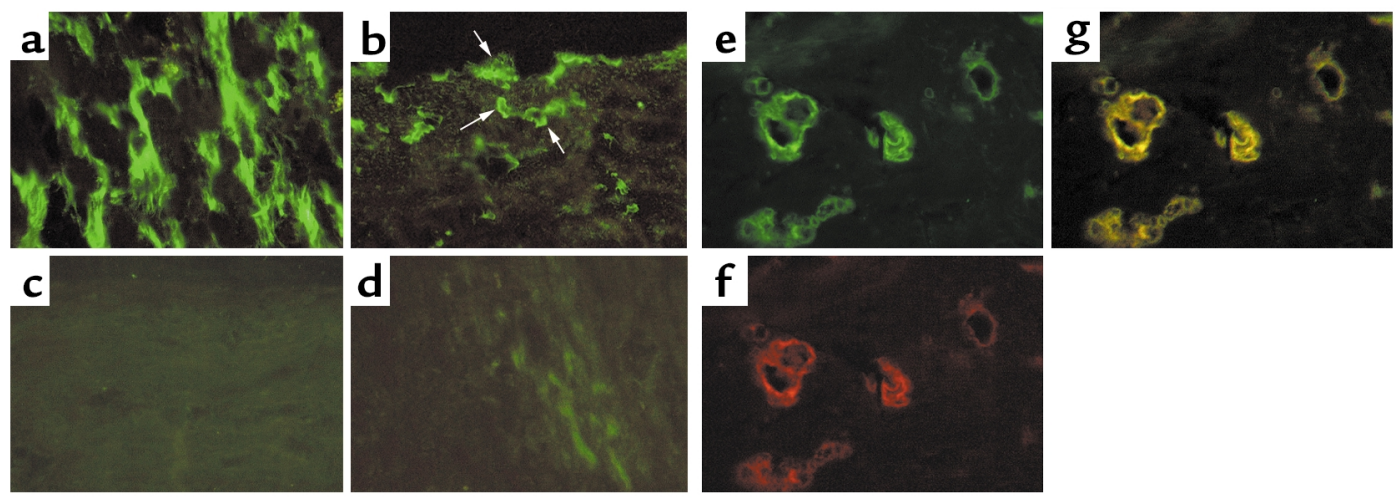

Figure 8

Localization of activated STAT5 and p2 1 waf in atherosclerotic plaques. (a-d) Activated STAT5. Specimens obtained from atherosclerotic lesions ( $\mathbf{a}$ and $\mathbf{b}$ ) or from nonatheromatous carotid artery ( $\mathbf{c}$ and $\mathbf{d}$ ) were subjected to immunofluorescent microscopy with an anti-phospho-STAT5 antibody. $\mathbf{a}$ and $\mathbf{d}$ show media; $\mathbf{b}$ and $\mathbf{c}$ show intima. Arrows indicate intimal neovessel and endothelial cells lining the luminal side. Specimens from atherosclerotic lesions were subjected to immunofluorescent microscopy with an anti-p21 waf antiserum (e) or with an anti-CD105 antiserum (f). Protein colocalization is shown in (g). 
ated with a significant impairment in adaptive vascular growth of both capillary-like tube vessels and collateral vessels (51-53). The observation that, through a STAT5B/p21 $1^{\text {waf }}$-mediated pathway, dm-LDL can affect the ability of endothelial cells to progress in the cell cycle adds further insight into the molecular mechanisms involved in the impaired vasculature remodeling in diabetes. Moreover, the evidence that activated STAT5 and p $21^{\text {waf }}$ were highly expressed in endothelial cells lining both the luminal side of the plaque and/or the intimal neovessels supports the possibility that a similar mechanism may be operative in vivo.

In conclusion, the results presented here demonstrate that the natural plasma constituent LDL, from type 2 diabetic patients, can maintain endothelial cells in a quiescent state in G1 through STAT5B-mediated p $21^{\text {waf }}$ expression. Moreover, the presence of a positive immunoreactivity for activated STAT5 and $\mathrm{p} 21^{\text {waf }}$ in intraplaque neovessels supports the possibility that induction of STAT5-dependent genes may exert substantial atherogenic effects on the vessel wall, and specifically, may account for the deranged adaptive vascular growth observed in this pathological condition. Finally, the recent observation that NF- $\mathrm{KB}$ and STAT5 regulate the expression of the same gene in T cells (54) raises the possibility that these transcriptional factors may also exert concerted effects on atherogenesis-related genes. However, further studies are required to elucidate the in vivo role of the STAT5 regulatory system.

\section{Acknowledgments}

We thank P. Defilippi for her helpful advice, and A. Miyajima for providing us with the $\triangle$ STAT5 constructs. This work was supported by grants from the Italian Association for Cancer Research (to G. Camussi, M.F. Brizzi, and L. Pegoraro), from the Ministero dell'Università e della Ricerca Scientifica e Tecnologica (to L. Pegoraro), and from the Consiglio Nazionale delle Ricerche: Targeted Projet on Biotechnology (to G. Camussi).

1. Ross, R. 1993. The pathogenesis of atherosclerosis: a perspective for the 1990s. Nature. 362:801-809.

2. King, G., and Brownlee, M. 1996. The cellular and molecular mechanism of diabetic complication. Endocrinol. Metab. Clin. North Am. 25:255-270.

3. Kannel, W.B., and McGee, D.L. 1979. Diabetes and cardiovascular disease: the Framingham study. JAMA. 241:2035-2038.

4. Penn, M.S., and Chisolm, G.M. 1994. Oxidized lipoproteins, altered cell function and atherosclerosis. Atherosclerosis. 108(Suppl.):S21-S29.

5. Lyons, T.J., and Jenkins, A.J. 1997. Lipoprotein glycation and its metabolic consequences. Curr. Opin. Lipidol. 8:174-180.

6. Shen, M.M., Krauss, R.M., Lindgren, F.T., and Forte, T.M. 1981. Heterogeneity of serum low density lipoproteins in normal human subjects. $J$. Lipid Res. 22:236-244.

7. Howard, B.V. 1994. Lipoprotein metabolism in diabetes. Curr. Opin. Lipidol. 5:216-220.

8. Collins, T., and Cybulsky, M.I. 2001. NF-кB: pivotal mediator or innocent bystander in atherogenesis? J. Clin. Invest. 107:255-264.

9. Ihle, J.N., and Kerr, I.M. 1995. Jaks and Stats in signaling by the cytokine receptor superfamily. Trends Genet. 11:69-74.

10. Mui, A.L., Wakao, H., O'Farrell, A.M., Harada, N., and Miyajima, A. 1995. Interleukin-3, granulocyte-macrophage colony stimulating factor and interleukin-5 transduce signals through two STAT5 homologs. EMBOJ. 14:1166-1175

11. Mui, A.L., Wakao, H., Kinoshita, T., Kitamura, T., and Miyajima, A. 1996. Suppression of interleukin-3-induced gene expression by a C-terminal truncated Stat5: role of Stat5 in proliferation. EMBO J. 15:2425-2433.

12. Morgan, D.O. 1995. Principles of CDK regulation. Nature. 374:131-134.
13. El-Deiry, W.S., et al. 1993. WAF1, a potential mediator of p53 tumor suppression. Cell. 75:817-825.

14. Harper, J.W., Adami, G.R., Wei, N., Keyomarsi, K., and Elledge, S.J. 1993. The $\mathrm{p} 21 \mathrm{Cdk}$-interacting protein Cip 1 is a potent inhibitor of $\mathrm{G} 1 \mathrm{cyclin}-$ dependent kinases. Cell. 75:805-816.

15. Waga, S., Hannon, G.J., Beach, D., and Stillman, B. 1994. The p21 inhibitor of cyclin-dependent kinases controls DNA replication by interaction with PCNA. Nature. 369:574-578.

16. Luo, Y., Hurwitz, J., and Massague, J. 1995. Cell-cycle inhibition by independent CDK and PCNA binding domains in p21Cip1. Nature. 375:159-161.

17. Chen, J., Jackson, P.K., Kirschner, M.W., and Dutta, A. 1995. Separate domains of p21 involved in the inhibition of Cdk kinase and PCNA. Nature. 374:386-388.

18. Datto, M.B., et al. 1995. Transforming growth factor beta induces the cyclin-dependent kinase inhibitor p21 through a p53-independent mechanism. Proc. Natl. Acad. Sci. USA. 92:5545-5549.

19. Mandal, M., Bandyopadhyay, D., Goepfert, T.M., and Kumar, R. 1998. Interferon-induces expression of cyclin-dependent kinase-inhibitors $\mathrm{p}^{2} 1^{\mathrm{WAF} 1}$ and $\mathrm{p}^{2} 7^{\mathrm{Kip} 1}$ that prevent activation of cyclin-dependent kinase by CDK-activating kinase (CAK). Oncogene. 16:217-225.

20. Polyak, K., et al. 1994. p27Kip1, a cyclin-Cdk inhibitor, links transforming growth factor-beta and contact inhibition to cell cycle arrest. Genes Dev. 8:9-22.

21. Akimoto, S., Mitsumata, M., Sasaguri, T., and Yoshida, Y. 2000. Laminar shear stress inhibits vascular endothelial cell proliferation by inducing cyclin-dependent kinase inhibitor p21(Sdi1/Cip1/Waf1). Circ. Res. 86:185-190.

22. Matsumura, I., et al. 1997. Thrombopoietin-induced differentiation of a human megakaryoblastic leukemia cell line, CMK, involves transcriptional activation of 21 (WAF1/Cip1) by STAT5. Mol. Cell. Biol. 17:2933-2943.

23. Adams, M.R., et al. 2000. Atherogenic lipids and endothelial dysfunction: mechanisms in the genesis of ischemic syndromes. Annu. Rev. Med. 51:149-167.

24. Redgrave, T.G., and Carlson, L.A. 1979. Changes in plasma very low density and low density lipoprotein content, composition, and size after a fatty meal in normo- and hypertriglyceridemic man. J. Lipid. Res. 20:217-229.

25. Lynch, S.M., and Frei, B. 1993. Mechanisms of copper- and iron- dependent oxidative modification of human low density lipoprotein. J. Lipid Res. 34:1745-1753.

26. Stocks, J., Nanjee, M.N., and Miller, N.E. 1998. Analysis of high density lipoproteins by capillary zone and capillary SDS gel electrophoresis. J. Lipid Res. 39:218-227.

27. Stocks, J., and Miller, N.E. 1998 Capillary electrophoresis to monitor the oxidative modification of low density lipoproteins. J. Lipid Res. 39:1305-1309.

28. Cruzado, I.D., Cockrill, C., McNeal, C.J., and Macfarlane, R.D. 1998. Characterization and quantitation of apolipoprotein B-100 by capillary electrophoresis. J. Lipid Res. 39:205-217.

29. Brizzi, M.F., et al. 1993. Interleukin-3 stimulates proliferation and triggers endothelial leukocyte adhesion molecule 1 gene activation of human endothelial cells. J. Clin. Invest. 91:2887-2892.

30. Brizzi, M.F., et al. 1999. Integrin-mediated adhesion of endothelial cells induces JAK2 and STAT5A activation; role in the control of c-fos gene expression. Mol. Biol. Cell. 10:3463-3471.

31. Sadowski, H.B., and Gilman, M.Z. 1993. Cell-free activation of a DNAbinding protein by epidermal growth factor. Nature. 362:78-93.

32. Brizzi, M.F., Rossi, P.R., Rosso, A., Avanzi, G.C., and Pegoraro, L. 1995 Transcriptional and post-transcriptional regulation of granulocytemacrophage colony-stimulating factor production in human growth factor dependent M-07e cells. Br. J. Haematol. 90:258-265.

33. Gariglio, P., Bellard, M., and Chambon, P. 1981. Clustering of RNA polymerase $B$ molecules in the $5^{\prime}$ moiety of the adult betaglobin gene of hen erythrocytes. Nucleic Acid Res. 9:2589-2598.

34. Lu, B., et al. 2001. Enhanced sensitivity of insulin-resistant adipocytes to vanadate is associated with oxidative stress and decreased reduction of vanadate (+5) to vanadyl (+4). J. Biol. Chem. 276:35589-35598.

35. Deshpande, S.S., Angkeow, P., Huang, J., Ozaki, M., and Irani, K. 2000. Rac1 inhibits TNF-alpha-induced endothelial cell apoptosis: dual regulation by reactive oxygen species. FASEB J. 14:1705-1714.

36. Brizzi, M.F., et al. 2001. Interleukin-3 stimulates migration and proliferation of vascular muscle cells. A potential role in atherogenesis. Circulation. 103:549-554.

37. Jenkins, A.J., et al. 2000. Native and modified LDL activate extracellular signal-regulated kinases in mesangial cells. Diabetes. 49:2160-2169.

38. Krieger, M. 1997. The other side of scavenger receptors: pattern recognition for host defense. Curr. Opin. Lipidol. 8:275-280.

39. Schmidt, A.M., and Stern, D.M. 2000. RAGE: a new target for the prevention and treatment of the vascular and inflammatory complications of diabetes. Trends Endocrinol. Metab. 11:368-375. 
40. Napoli, C., de Nigris, F., and Palinski, W. 2001. Multiple role of reactive oxygen species in the arterial wall. J. Cell. Biochem. 82:674-682.

41. Maziere, C., et al. 1999. Oxidized LDL activates STAT1 and STAT3 transcription factors: possible involvement of reactive oxygen species. FEBS Lett. 448:49-52.

42. Bubenik, J., Perlmann, P., Helmstein, K., and Moberger, G. 1970. Cellular and humoral immune responses to human urinary bladder carcinomas. Int. J. Cancer. 5:310-319.

43. Ricard, I., Payet, M.D., and Dupuis, G. 1998. VCAM-1 is internalized by a clathrin-related pathway in human endothelial cells but its alpha 4 beta 1 integrin counter-receptor remains associated with the plasma membrane in human T lymphocytes. Eur. J. Immunol. 28:1708-1718.

44. Liu, J., et al. 1999. Angiogenesis activators and inhibitors differentially regulate caveolin-1 expression and caveolae formation in vascular endothelial cells. Angiogenesis inhibitors block vascular endothelial growth factor-induced down-regulation of caveolin-1.J. Biol. Chem. 274:15781-15785.

45. Escargueil-Blanc, I., et al. 1998. Apoptosis and activation of the sphingomyelin-ceramide pathway induced by oxidized low density lipoproteins are not causally related in ECV-304 endothelial cells. J. Biol. Chem. 273:27389-27395

46. Polyak, K., et al. 1994. Cloning of p27Kip1, a cyclin-dependent kinase inhibitor and a potential mediator of extracellular antimitogenic signals.
Cell. 78:59-66

47. Toyoshima, H., and Hunter, T. 1994. p27, a novel inhibitor of G1 cyclinCdk protein kinase activity, is related to 21 . Cell. 78:67-74.

48. Matsumura, I., et al. 1999. Transcriptional regulation of the cyclin D1 promoter by STAT5: its involvement in cytokine-dependent growth of hematopoietic cells. EMBOJ. 18:1367-1377.

49. Austin, M.A., King, M.C., Vranizan, K.M., and Krauss, R.M. 1990. Atherogenic lipoprotein phenotype. A proposed genetic marker for coronary heart disease risk. Circulation. 82:495-506.

50. Isner, J.M. 1994. Vascular remodeling. Circulation. 89:2937-2941.

51. Chen, C.H., et al. 1997. Inhibitory effects of hypercholesterolemia and oxLDL on angiogenesis-like endothelial growth in rabbit aortic explants. Essential role of basic fibroblast growth factor. Arterioscler. Thromb. Vasc. Biol. 17:1303-1312.

52. Abaci, A., et al. 1999. Effect of diabetes mellitus on formation of coronary collateral vessels. Circulation. 99:2239-2242.

53. Chen, C.H., et al. 2000. Oxidized low-density lipoproteins inhibit endothelial cell proliferation by suppressing basic fibroblast growth factor expression. Circulation. 101:171-177.

54. Musikacharoen, T., Matsuguchi, T., Kikuchi, T., and Yoshikai, Y. 2001. NFkappa B and STAT5 play important roles in the regulation of mouse Tolllike receptor 2 gene expression. J. Immunol. 166:4516-4524. 